\title{
A Review of the Recent Advances in Ultra-High Field Magnetic Resonance Imaging
}

\author{
Mehran Shams Kondori
}

\begin{abstract}
Recent advances in ultra-high field magnetic resonance imaging has addressed substantial technological challenges in both hardware and software. These challenges, including transmit field inhomogeneity, primarily are due to the onset of far-field effects at the resonance frequencies at 7Tesla and 10.5Tesla MRI which becomes more demanding at higher field strengths. The advent of parallel imaging techniques in reception (multi-channel radio-frequency arrays), transmission (parallel transmit or pTx), and reconstruction (especially using deep learning models) has been an effort to address such challenges. Here, the most recent notable advances in MRI in both hardware and software fronts and their implications for human brain neuroscience applications are reviewed.
\end{abstract}

\section{INTRODUCTION}

$\mathbf{M}$ AGNETIC Resonance Image (MRI) is a non-invasive medical imaging modality used extensively in clinical and research settings to image the interiors of human body. Today, the strength of the static magnetic field, $B_{0}$ used in the MRI scanner is the most prominent specification of the machinery. Clinical MR scanners with $B_{0}=1.5$ Tesla $(\mathrm{T})$ are the most common followed by $B_{0}=3 \mathrm{~T}$ machines [1]. The ${ }^{1} \mathrm{H}$ Larmor frequency at these field strengths $(128 \mathrm{MHz}$ at 3T) is significantly less than the frequency at ultra-high field (UHF) MRI machines, defined as those with $B_{0}>3 \mathrm{~T}$ [2]. The increased frequency at ultra-high field scanners results in smaller wave-length, especially inside the human body. The smaller wavelength means that the electromagnetic regime of the antenna used to transmit or receive signal involves far-field effects which cause field inhomogeneity and its consequent challenges. Of course, the higher the resonant frequency (e.g. $447 \mathrm{MHz}$ at $10.5 \mathrm{~T}$ ), the shorter the wavelength is and the more exacerbated these challenges are [3].

One of the primary avenues to deal with these challenges is parallel imaging [4]. In the context of MRI, parallel imaging are methods such as SMASH [5], SENSE [6] and GRAPPA [7] that use the independent information of receiver channels from

Department of Mechanical Engineering, Virginia Tech, Blacksburg, VA 24060. E-mail: mehran@vt.edu. a receive array to allow for compensation of the artifacts caused by subsampling the frequency domain ( $k$-space) signal. Such artifacts include aliasing (which is a consequent of the sub-Nyquist sampling rate) and noise amplification, which happens as a byproduct of the common reconstruction techniques such as GRAPPA. GRAPPA is a parallel imaging reconstruction technique that uses the redundant information of the receive arrays to solve for the missing signal ( $k$-space) lines and then, reconstruct images using Fourier transform and combine the images from various channels through some algorithm, such as root-sum-of-squares. GRAPPA reconstruction characteristically enhances the noise of the image. It should be noted that compressed sensing [8] techniques are a separate set of methods usually not listed as traditional parallel imaging methods although they do rely on the compressible nature of MRI images.

Another challenge arising from the shorter wavelength is electromagnetic field inhomogeneity [9], [10] which is often tackled either by parallel transmit (pTx) using multi-channel transmitter arrays (radio-frequency antennae often composed of several dipole or LC tank loop elements) or shimming the transmit magnetic field $B_{1}^{+}$(where static $B_{1}^{+}$shimming is adjusting the phase of various radio-frequency pulses on each of the channels so as to improve homogeneity or efficiency of the combined $B_{1}^{+}$field from several channels) [11], [12]. Furthermore, the analysis of the specific absorption rate (SAR) [13] which is necessary for safety validation of transmitters (to ensure that the power deposited in the human tissue is less than the limits set by standards and regulatory guidelines) becomes increasingly challenging as the field inhomogeneity increases. This is because the difference between the expected electromagnetic fields (calculated using full-wave electromagnetic circuit cosimulations) and experimentally measured fields becomes more complex due to such field inhomogeneities. Another factor that contributes to these field mismatches is deviations in manual construction of radiofrequeuncy (RF) coils from the design which become 
more electromagnetically significant and problematic at higher frequencies (shorter wavelengths).

In the next section, some of the recent advances in MRI technology that address these and additional challenges are reviewed. While not an exhaustive list, the following sections point to the leading studies in pioneering research institutes in the field.

\section{HARDWARE ADVANCES}

When designing receive arrays, MRI researchers have two performance metrics in mind: signal-to-noise ratio (SNR) and noise correlation (the crosstalk of different receive elements of a receive array) [14]-[16]. In terms of SNR, increasing the static field strength (which is a specification of the MR scanner) and increasing the number of channels in the receive array (which is a design parameter set by the RF coil researcher) can lead to increases in SNR [4], [17], [18]. Both of these avenues, especially increasing the number of receive channels, however, seems to have complications. With increased field strength, the wavelength shortens and all of the challenges discussed previously arise. With increased number of channels, each receive element becomes smaller and its coupling into the sample (conductive loading of the element) becomes smaller leading to the risk of greater electronic noise dominance. Electronic noise dominance cannot be mitigated in the postprocessing steps and therefore reduces SNR. In order to avoid this effect, RF coil designers try to keep the receive array as form-fitting as possible.

The more intractable challenge in design and construction of receive arrays is the noise correlation [16], [19]. Many decoupling strategies (methods to mitigate noise correlation) have been proposed in the past decades. Roemer et al. demonstrated that overlap decoupling effectively causes electric coupling to cancel magnetic coupling of loop elements [20]. Inductive and transformer decoupling as well as decoupling networks were used extensively in construction of various receive arrays for different field strengths [14]-[16], [21]-[24]. Most recently, the concept of self-decoupling was introduced [25]. Other researchers [26] brilliantly developed the self-decoupling concept to simplify the design of receive arrays and also demonstrated experimentally that self-decoupling provides an SNR advantage compared to overlap-decoupling. The latter stands as a much anticipated breakthrough that obviates the complexities of building high density receive arrays. Another challenge in building receive arrays is the cable management problem. The sheer amount of copper cables in a high density receive

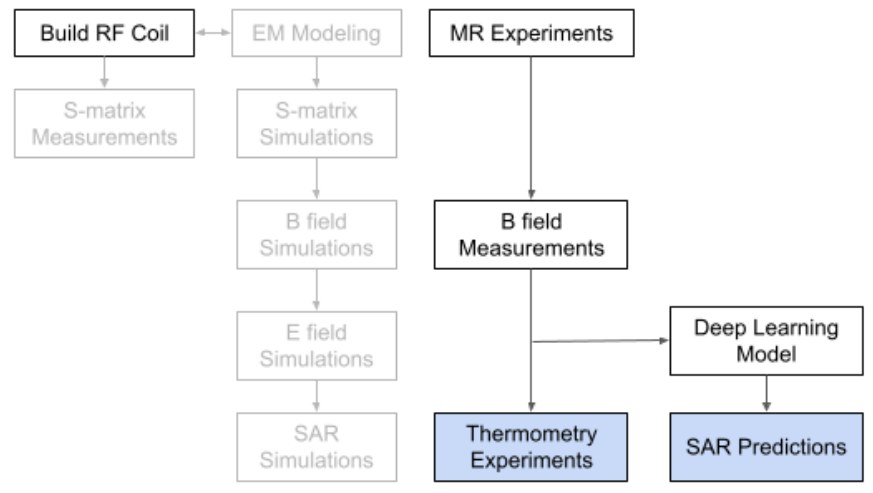

Fig. 1. The pipeline of RF coil safety validation, showing potential for integrating deep learning models.

array and the current induced on these cables can interfere with the transmit field [27], [28]. This challenge still stands to be resolved effectively.

Another promising line of research to improve signal-tonoise ratio is the efforts studying the effects of shielding the RF coil [29] or using high permittivity materials [30] in combination with RF receive arrays to increase SNR. These studies have shown improvements in simulations and have a promising prospect once they are fully implemented experimentally. These increases in signal-to-noise ratio (such as reported in [26]) are substantial progress towards empowering neuroscience research applications targeting human brain [31].

\section{SOFTWARE ADVANCES}

A second set of advances includes both methods in safety validation and reconstruction. Most of these techniques are made possible by the integration of data driven methods into the pipeline.

Safety validation of MR transmitters can be an intricate process. While at lower frequencies (1.5T to $7 \mathrm{~T}$ or $298 \mathrm{MHz}$ ) the regulatory bodies seem to have accepted the existing coil technologies as safe and do not require meticulous safety validation of derivative coil designs, at ultra-high field (10.5T / 447MHz) detailed safety validation is still a necessity [3]. These safety validations often involve full-wave electromagnetic simulations [24] which try to replicate the scatter matrix parameters measured with the physical coil on the bench [13]. It should be noted that such electromagnetic software are still complementary to experimental verification [33]-[37]. The $\mathrm{S}$ parameter match is often presumed to result in a match between the simulated and experimentally measured magnetic fields produced by the coil (see Figrue 1). However, this is not a guaranteed outcome as the parasitic reactances that inevitably 


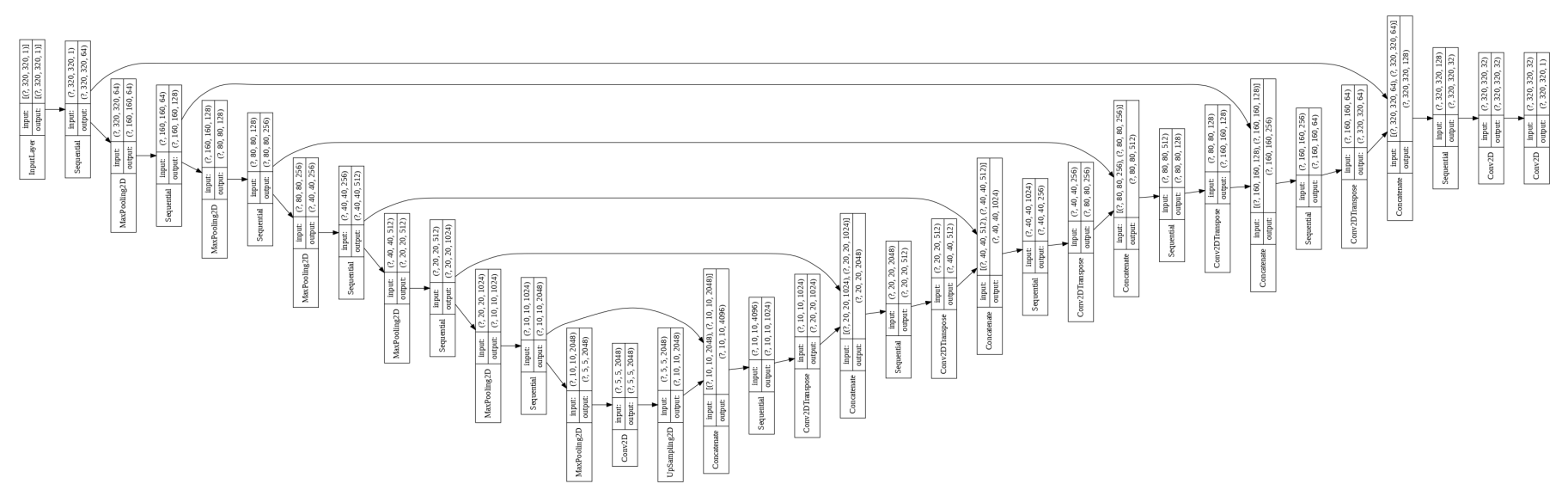

Fig. 2. The architecture of a representative UNET printed using Tensorflow [32].

arise in the physical coil are often prohibitively difficult to replicate in simulation. While lower frequency coil models can be forgiving, at ultra-high field the shorter wavelength demands a more accurate model. Such deviations combined with the interactions of the transmit elements with each other and with receive elements can cause significant differences between the simulated and measured field, barring the path to accurate SAR calculations. These and similar challenges are motivating the use of data-driven models to predict the electromagnetic field of an RF coil. As indicated in Figure 1, in future deep learning-based models can be trained to predict SAR maps reliably given the experimentally measured magnetic fields as input, obviating the current excruciating need for replication of experimental fields using often inefficient full-wave simulators ${ }^{1}$.

Recently, there have been several efforts in parallel that have tried to tackle these obstacle. In [38], the authors propose a method which is an effort to integrate the ultimate field match back into the earlier steps of the simulation. They formulate a loss function that includes both the S-parameter match and the image domain field match and is therefore an effort to bridge over the obstacle discussed before. More interestingly, in [39], the authors present a data driven model that, once trained, can facilitate the safety validation process.

Beyond the safety validation effort, another area of MRI that has seen incredible developments in the recent years is reconstruction. Conventional parallel imaging techniques such as SMASH [5]-[7], [40] and their derivatives and those techniques that evolved from these seminal works each have their advantages and disadvantages. In short, they generally rely

\footnotetext{
${ }^{1}$ While such full-wave electromagnetic simulators are efficient for small simulations, simulating a multi-channel antenna array can take time on the order of several days or a week, especially if a complicated model of human body is involved. These lengthy simulations make iterative design inefficient.
}

on the extra information from independent receive channels to compensate for the information lost to undersampling the signal. This reduces image acquisition time but comes with several costs, most notably increased noise in the reconstructions. Compressed sensing [8] techniques are not an exception either. All of these techniques provide advantages: essentially reduced acquisition time, but each has its drawbacks. Recently, researchers have started to look at deep learning models to complement and improve these conventional methods in an evolutionary or revolutionary way.

Unrolling techniques [41]-[44] are one class of such deep learning based methods. The primary advantage of unrolling techniques compared to other deep learning based algorithms is that unrolled networks share parameters, therefore the number of trainable parameters of these unrolled networks is much less than what is typical in other techniques, even with a simple UNET [45] (see Figrue 2). This allows the network to be trained more efficiently, with more epochs given the same amount of computational resources (gpuhours). The works of [41], [42] were remarkable advances in several ways, most prominently the self-supervised learning work which splits the already undersampled $k$-space into two disjoint subsets and basically uses one subset for training and the other subset for hyper-parameter optimization much in the same way that $\mathrm{k}$-fold cross-validation works. There are other promising developments in the reconstruction front as well. The fastMRI dataset [46], [47] and other public MR datasets have attracted a lot of attention from both MR researchers and computer scientists as a benchmark that allows consistent performance comparison across methods and hence, replicable progress. Some of the works that followed the fastMRI challenge [48]-[51] were substantial steps forward in applying data driven models to various MR-related tasks. There is broad 
interest in the MR research community to use various deep learning techniques to tackle the reconstruction task. These techniques have often outperformed the conventional parallel imaging reconstruction methods [50], [52]-[58]. It is inevitable that, after further extensive validation of these techniques in terms of anatomical fidelity, they will be integrated into the clinical MR systems (e.g. [59], [60]) as they provide clear advantages in terms of reconstruction time (especially compared to iterative techniques, such as ISTA [61] or regularized GRAPPA), structural similarity and peak signal-to-noise ratio.

\section{CONCLUSION}

In this review, we have discussed those notable advances in the ultra-high field MRI technology that aim to address the long-standing obstacles impeding the progress of researchers. Issues arising from the higher frequency / shorter wavelength causing a far-field regime in the human body, such as field inhomogeneity and the resulting complexities of safety validations, were discussed. Furthermore, recent advances that tackle these challenges, specifically in RF array design technology, safety validation, and reconstruction were reviewed. The papers mentioned, considered together, establish a perspective of the milestone achievements in the ultra-high field MR landscape which set the stage for consequential breakthroughs in the field.

\section{REFERENCES}

[1] S. O. Dumoulin, A. Fracasso, W. van der Zwaag, et al., "Ultrahigh field MRI: Advancing systems neuroscience towards mesoscopic human brain function," NeuroImage, vol. 168, pp. 345-357, Mar. 2018.

[2] K. Ugurbil, "Magnetic Resonance Imaging at Ultrahigh Fields," IEEE Transactions on Biomedical Engineering, vol. 61, no. 5, pp. 1364 1379, May 2014.

[3] K. Uğurbil, "Imaging at ultrahigh magnetic fields: History, challenges, and solutions," NeuroImage, vol. 168, pp. 7-32, Mar. 2018.

[4] F. Wiesinger, P.-F. Van de Moortele, G. Adriany, et al., "Parallel imaging performance as a function of field strength - An experimental investigation using electrodynamic scaling," Magnetic Resonance in Medicine, vol. 52, no. 5, pp. 953-964, Nov. 2004.

[5] D. K. Sodickson and W. J. Manning, "Simultaneous acquisition of spatial harmonics (SMASH): Fast imaging with radiofrequency coil arrays," Magnetic Resonance in Medicine, vol. 38, no. 4, pp. 591-603, Oct. 1997.

[6] K. P. Pruessmann, M. Weiger, M. B. Scheidegger, et al., "SENSE: Sensitivity encoding for fast MRI," Magnetic Resonance in Medicine, vol. 42, no. 5, pp. 952-962, Nov. 1999.

[7] M. A. Griswold, P. M. Jakob, R. M. Heidemann, et al., "Generalized autocalibrating partially parallel acquisitions (GRAPPA)," Magnetic Resonance in Medicine, vol. 47, no. 6, pp. 1202-1210, Jun. 2002.

[8] D. Donoho, "Compressed sensing," IEEE Transactions on Information Theory, vol. 52, no. 4, pp. 1289-1306, Apr. 2006.

[9] G. Adriany, P.-F. F. Van De Moortele, F. Wiesinger, et al., "Transmit and receive transmission line arrays for 7 tesla parallel imaging," Magnetic Resonance in Medicine, vol. 53, no. 2, pp. 434-445, Feb. 2005.

[10] P.-F. F. Van de Moortele, C. Akgun, G. Adriany, et al., "B1 destructive interferences and spatial phase patterns at $7 \mathrm{~T}$ with a head transceiver array coil," Magnetic Resonance in Medicine, vol. 54, no. 6, pp. 15031518, Dec. 2005.
[11] G. Adriany, J. Ritter, T. Vaughan, et al., "Experimental verification of enhanced B1 Shim performance with a Z-encoding RF coil array at 7 tesla," Proc. Intl. Soc. Mag. Reson. Med., vol. 3831, 2010.

[12] P. F. Van de Moortele, C. Snyder, L. DelaBarre, et al., "Calibration Tools for RF Shim at Very High Field with Multiple Element RF Coils : from Ultra Fast Local Relative Phase to Absolute Magnitude B1+ Mapping," Proc. Intl. Soc. Mag. Reson. Med. 15, p. 1676, 2007.

[13] T. M. Fiedler, M. E. Ladd, and A. K. Bitz, "SAR Simulations \& Safety," NeuroImage, no. March, 2017.

[14] B. Keil, J. N. Blau, S. Biber, et al., "A 64-channel 3T array coil for accelerated brain MRI," Magnetic Resonance in Medicine, vol. 70, no. 1, pp. 248-258, Jul. 2013.

[15] B. Keil, "Construction of Receive Arrays," Proc. Intl. Soc. Mag Reson. Med., vol. 21, pp. 1-10, 2013.

[16] B. Keil and L. L. Wald, "Massively parallel MRI detector arrays," Journal of Magnetic Resonance, vol. 229, pp. 75-89, Apr. 2013.

[17] F. Wiesinger, P. Boesiger, and K. P. Pruessmann, "Electrodynamics and ultimate SNR in parallel MR imaging," Magnetic Resonance in Medicine, vol. 52, no. 2, pp. 376-390, Aug. 2004.

[18] F. Wiesinger, N. De Zanche, and K. P. Pruessmann, "Approaching Ultimate SNR with Finite Coil Arrays," Proceedings of the International Society for Magnetic Resonance in Medicine 13, vol. 13, no. 1, p. 672,2005

[19] X. Cao, E. Fischer, O. Gruschke, et al., "The noise factor of receiver coil matching networks in MRI," Magnetic Resonance Imaging, vol. 37, pp. 252-259, Apr. 2017.

[20] P. B. Roemer, W. A. Edelstein, C. E. Hayes, et al., "The NMR phased array," Magnetic Resonance in Medicine, vol. 16, no. 2, pp. 192-225, Nov. 1990.

[21] G. Shajan, J. Hoffmann, J. Budde, et al., "Design and evaluation of an RF front-end for 9.4 T human MRI," Magnetic Resonance in Medicine, vol. 66, no. 2, pp. 594-602, Aug. 2011.

[22] G. Shajan, M. Kozlov, J. Hoffmann, et al., "A 16-channel dual-row transmit array in combination with a 31-element receive array for human brain imaging at 9.4 T," Magnetic Resonance in Medicine, vol. 71, no. 2, pp. 870-879, Feb. 2014.

[23] G. Shajan, J. Hoffmann, G. Adriany, et al., "A 7T Head Coil with 16-channel dual-row transmit and 31-channel receive for pTx applications," in 24th Annual Meeting and Exhibition of the International Society for Magnetic Resonance in Medicine, 2016.

[24] G. Adriany, J. Radder, N. Tavaf, et al., "Evaluation of a 16-Channel Transmitter for Head Imaging at 10.5T," in 2019 International Conference on Electromagnetics in Advanced Applications (ICEAA), IEEE, Sep. 2019, pp. 1171-1174.

[25] X. Yan, J. C. Gore, and W. A. Grissom, "Self-decoupled radiofrequency coils for magnetic resonance imaging," Nature Communications, vol. 9, no. 1, p. 3481, Dec. 2018

[26] N. Tavaf, R. L. Lagore, S. Jungst, et al., "A Self-Decoupled 32 Channel Receive Array for Human Brain Magnetic Resonance Imaging at 10.5T," arxiv preprint arXiv: 2009.07163v2, Sep. 2020.

[27] D. Seeber, J. Jevtic, and A. Menon, "Floating shield current suppression trap," Concepts in Magnetic Resonance, vol. 21B, no. 1, pp. 26-31, Apr. 2004.

[28] J. Brown and B. Whitlock, "Common-Mode to Differential-Mode Conver-sion in Shielded Twisted-Pair Cables (Shield-Current-Induced Noise)," 114th AES Convention in Amsterdam, p. 5747, 2003.

[29] B. Zhang, G. Adriany, A. Grant, et al., "Shielding Effects on Signalto-Noise Ratio at Ultra-High Field MRI," International Society of Magnetic Resonance in Medicine, p. 0752, 2020.

[30] G. Carluccio, B. Zhang, R. Lattanzi, et al., "Effects of Parallel Imaging Acceleration on SNR Improvement with a High-Permittivity Helmet Shaped Former at Different Field Strengths," International Society of Magnetic Resonance in Medicine, p. 1567, 2019.

[31] T. F. Budinger and M. D. Bird, "MRI and MRS of the human brain at magnetic fields of $14 \mathrm{~T}$ to $20 \mathrm{~T}$ : Technical feasibility, safety, and neuroscience horizons," NeuroImage, vol. 168, pp. 509-531, Mar. 2018.

[32] M. Abadi, A. Agarwal, P. Barham, et al., "TensorFlow: LargeScale Machine Learning on Heterogeneous Distributed Systems," arXiv:1603.04467v2, Mar. 2016.

[33] J. Radder, M. K. Woo, P. F. Van De Moortele, et al., "Optimization and simulation of a 16-channel loop and dipole array for head MRI applications at 10.5 Tesla," Proceedings of the 2017 19th International Conference on Electromagnetics in Advanced Applications, ICEAA 2017, pp. 1828-1831, 2017.

[34] M. K. Woo, R. L. Lagore, L. Delabarre, et al., "A 16-channel transceiver loop+dipole antennas head array for human head imaging 
at 10.5T," Proceedings of the 2017 19th International Conference on Electromagnetics in Advanced Applications, ICEAA 2017, pp. 16491652, 2017.

[35] J. W. Radder, S. Moeller, G. Adriany, et al., "Simulation of B1 efficiency in 64-channel phased head arrays at 7T and 10.5T," Proc Intl Soc Mag Reson Med, vol. 25, no. 3, p. 4285, 2017.

[36] G. Giovannetti and G. Tiberi, "Radiofrequency magnetic resonance coils and communication antennas: Simulation and design strategies," Magnetic Resonance Imaging, vol. 44, pp. 1-7, Dec. 2017.

[37] H. Rahmaninejad, T. Pace, S. Bhatt, et al., "Co-localization and confinement of ectonucleotidases modulate extracellular adenosine nucleotide distributions," PLoS Computational Biology, vol. 16, no. 6, e1007903, Jun. 2020

[38] X. Li, J. W. Pan, N. I. Avdievich, et al., "Electromagnetic simulation of a 16-channel head transceiver at $7 \mathrm{~T}$ using circuit-spatial optimization," Magnetic Resonance in Medicine, no. December 2020, mrm.28672, Feb. 2021.

[39] E. Meliadò, A. Raaijmakers, A. Sbrizzi, et al., "A deep learning method for image-based subject-specific local SAR assessment," Magnetic Resonance in Medicine, mrm.27948, Sep. 2019.

[40] M. Blaimer, F. Breuer, M. Mueller, et al., "SMASH, SENSE, PILS, GRAPPA," Topics in Magnetic Resonance Imaging, vol. 15, no. 4, pp. 223-236, Aug. 2004.

[41] S. A. H. Hosseini, B. Yaman, S. Moeller, et al., "Dense Recurrent Neural Networks for Accelerated MRI: History-Cognizant Unrolling of Optimization Algorithms," Dec. 2019.

[42] B. Yaman, S. A. H. Hosseini, S. Moeller, et al., "Self-supervised learning of physics-guided reconstruction neural networks without fully sampled reference data," Magnetic Resonance in Medicine, mrm.28378, Jul. 2020

[43] F. Knoll, K. Hammernik, C. Zhang, et al., "Deep-Learning Methods for Parallel Magnetic Resonance Imaging Reconstruction: A Survey of the Current Approaches, Trends, and Issues," IEEE Signal Processing Magazine, vol. 37, no. 1, pp. 128-140, Jan. 2020.

[44] D. Liang, J. Cheng, Z. Ke, et al., "Deep Magnetic Resonance Image Reconstruction: Inverse Problems Meet Neural Networks," IEEE Signal Processing Magazine, vol. 37, no. 1, pp. 141-151, Jan. 2020.

[45] O. Ronneberger, P. Fischer, and T. Brox, "U-Net: Convolutional Networks for Biomedical Image Segmentation," Lecture Notes in Computer Science (including subseries Lecture Notes in Artificial Intelligence and Lecture Notes in Bioinformatics), vol. 9351, pp. 234 241, May 2015

[46] J. Zbontar, F. Knoll, A. Sriram, et al., "fastMRI: An Open Dataset and Benchmarks for Accelerated MRI," Nov. 2018.

[47] F. Knoll, J. Zbontar, A. Sriram, et al., "fastMRI: A Publicly Available Raw k-Space and DICOM Dataset of Knee Images for Accelerated MR Image Reconstruction Using Machine Learning," Radiology: Artificial Intelligence, vol. 2, no. 1, e190007, Jan. 2020.

[48] N. Pezzotti, S. Yousefi, M. S. Elmahdy, et al., "An Adaptive Intelligence Algorithm for Undersampled Knee MRI Reconstruction: Application to the 2019 fastMRI Challenge," Tech. Rep.

[49] F. Knoll, T. Murrell, A. Sriram, et al., "Advancing machine learning for MR image reconstruction with an open competition: Overview of the 2019 fastMRI challenge," Tech. Rep., 2020.

[50] N. Tavaf, A. Torfi, K. Ugurbil, et al., "GRAPPA-GANs for Parallel MRI Reconstruction," arXiv preprint arXiv:2101.03135, Jan. 2021.

[51] A. Torfi, R. A. Shirvani, Y. Keneshloo, et al., "Natural Language Processing Advancements By Deep Learning: A Survey," arXiv:2003.01200v3, Mar. 2020.

[52] G. Yang, S. Yu, H. Dong, et al., "DAGAN: Deep De-Aliasing Generative Adversarial Networks for Fast Compressed Sensing MRI Reconstruction," IEEE Transactions on Medical Imaging, vol. 37, no. 6, pp. 1310-1321, 2018.

[53] K. Hammernik, T. Klatzer, E. Kobler, et al., "Learning a Variational Network for Reconstruction of Accelerated MRI Data," Tech. Rep., 2017

[54] T. M. Quan, T. Nguyen-Duc, and W.-K. Jeong, "Compressed Sensing MRI Reconstruction Using a Generative Adversarial Network With a Cyclic Loss," IEEE Transactions on Medical Imaging, vol. 37, no. 6, pp. 1488-1497, Jun. 2018.

[55] A. Sriram, J. Zbontar, T. Murrell, et al., "End-to-End Variational Networks for Accelerated MRI Reconstruction," Tech. Rep.

[56] C. Min Hyun, H. Pyung Kim, S. Min Lee, et al., "Deep learning for undersampled MRI reconstruction," Tech. Rep., 2019.

[57] S. U. Dar, M. Yurt, M. Shahdloo, et al., "Prior-guided image reconstruction for accelerated multi-contrast mri via generative adversarial networks," IEEE Journal on Selected Topics in Signal Processing, vol. 14, no. 6, pp. 1072-1087, 2020.

[58] B. Murugesan, V. R. S, K. Sarveswaran, et al., "Recon-GLGAN: A Global-Local context based Generative Adversarial Network for MRI Reconstruction," Aug. 2019.

[59] C. Gros, A. Lemay, O. Vincent, et al., "ivadomed: A Medical Imaging Deep Learning Toolbox," Tech. Rep.

[60] A. S. Lundervold and A. Lundervold, An overview of deep learning in medical imaging focusing on MRI, May 2019.

[61] J. Zhang and B. Ghanem, "ISTA-Net: Interpretable OptimizationInspired Deep Network for Image Compressive Sensing," Proceedings of the IEEE Computer Society Conference on Computer Vision and Pattern Recognition, pp. 1828-1837, Jun. 2017. 